\title{
NÉRON-SEVERI GROUP OF A GENERAL HYPERSURFACE
}

\author{
VINCENZO DI GENNARO AND DAVIDE FRANCO
}

\begin{abstract}
In this paper we extend the well-known theorem of Angelo Lopez concerning the Picard group of the general space projective surface containing a given smooth projective curve, to the intermediate Néron-Severi group of a general hypersurface in any smooth projective variety.
\end{abstract}

Keywords: Noether-Lefschetz Theory, Néron-Severi group, Borel-Moore Homology, Monodromy representation, Isolated singularities, Blowing-up.

MSC2010: 14B05, 14C20, 14C21, 14C22, 14C25, 14C30, 14F43, 14F45, 14J70.

\section{INTRODUCTION}

A well-known result of Angelo Lopez [13, inspired by a previous work of Griffiths and Harris 11, provides a recipe for the computation of the Néron-Severi group $N S_{1}(S ; \mathbb{Z})$ of a general complex surface $S$ of sufficiently large degree in $\mathbb{P}^{3}$, containing a given smooth curve. For a smooth projective variety $X$, we define the $i$-th Néron-Severi group $N S_{i}(X ; \mathbb{Z})$ as the image of the cycle map $A_{i}(X) \rightarrow$ $H_{2 i}(X ; \mathbb{Z}) \cong H^{2(\operatorname{dim} X-i)}(X ; \mathbb{Z})([8, \S 19.1)$. This work was intended as an attempt to extend Lopez's result to the intermediate Néron-Severi group $N S_{\operatorname{dim} X / 2}(X ; \mathbb{Z})$ of a general hypersurface $X$, in any smooth projective variety. In the previous paper 4 . we already obtained a generalization, but only in the case of $\mathbb{Q}$-coefficients, i.e. only for $N S_{\operatorname{dim} X / 2}(X ; \mathbb{Q}):=N S_{\operatorname{dim} X / 2}(X ; \mathbb{Z}) \otimes_{\mathbb{Z}} \mathbb{Q}$. More precisely, in (4, Theorem 1.2), we proved the following:

Theorem 1.1. Let $Y \subset \mathbb{P}=\mathbb{P}(\mathbb{C})$ be a smooth projective variety of dimension $m+1=2 r+1$ and set $\mathcal{V}_{d}:=\operatorname{Im}\left(H^{0}\left(\mathbb{P}, \mathcal{O}_{\mathbb{P}}(d)\right) \rightarrow H^{0}\left(Y, \mathcal{O}_{Y}(d)\right)\right)$. Let $Z \subset Y$ be a closed subscheme of dimension $r$ contained in a regular sequence of smooth hypersurfaces $\bar{X} \in\left|\mathcal{V}_{d}\right|, G_{i} \in\left|\mathcal{V}_{d_{i}}\right|, 1 \leq i \leq r$, such that $d>d_{1}>\cdots>d_{r}$. Let $X \in\left|\mathcal{V}_{d}\right|$ be a very general hypersurface containing $Z$, so that $Z$ is a closed subscheme of the complete intersection $\Delta:=X \cap G_{1} \cap \cdots \cap G_{r}$,

$$
\Delta=Z \cup R=\left(\bigcup_{i=1}^{\rho} Z_{i}\right) \cup\left(\bigcup_{j=1}^{\sigma} R_{j}\right) .
$$

Assume that the vanishing cohomology of $X$ is not of pure Hodge type $\left(\frac{m}{2}, \frac{m}{2}\right)$. Denote by $H^{m}(X ; \mathbb{Z})_{\Delta}$ the subgroup of $H^{m}(X ; \mathbb{Z})$ generated by the components of $\Delta$, and by $H^{m}(X ; \mathbb{Z})_{\Delta^{-}}$the subgroup of $H^{m}(X ; \mathbb{Z})$ generated by $Z_{1}, \ldots, Z_{\rho}$, $R_{1}, \ldots, R_{\sigma-1}$. Then we have:

(1) $H^{m}(X ; \mathbb{Z})_{\Delta}$ is free of rank $\rho+\sigma$;

(2) $N S_{r}(X ; \mathbb{Q})=N S_{r+1}(Y ; \mathbb{Q}) \oplus H^{m}(X ; \mathbb{Q})_{\Delta^{-}}$. 
The aim of this paper is to improve previous Theorem 1.1, showing that:

Theorem 1.2.

$$
N S_{r}(X ; \mathbb{Z})=\left[N S_{r}(X ; \mathbb{Z}) \cap H^{m}(Y ; \mathbb{Z})\right] \oplus H^{m}(X ; \mathbb{Z})_{\Delta^{-}}
$$

We would like to stress that even though the main troubles in the proof of Theorem 1.2 come from the singularities of $\Delta$, such a result is not trivial even for smooth $\Delta$. Indeed, although in this case $\tilde{Y}:=B l_{\Delta}(Y)$ would be smooth, the strict transform $\tilde{X}:=B l_{\Delta}(X)$ would vary in a linear system which is not very ample on $\widetilde{Y}$. In fact, as it is proved in Proposition 2.12, this linear system contracts $\bigcap_{i=1}^{r} G_{i}$ to a point. Therefore, one cannot apply Lefschetz Hyperplane Theorem directly. Actually, it is our opinion that even for smooth $\Delta$ it would be difficult to avoid the arguments used in this note.

As explained in the body of the paper, the main technical point in the proof of Theorem 1.2 refers to the following Lefschetz-type problem:

Question 1. Let $G \subseteq \mathbb{P}$ be an irreducible, smooth projective variety of dimension $m=2 r \geq 2$, and fix a hypersurface $W \in\left|H^{0}\left(G, \mathcal{O}_{G}(d)\right)\right|(d \geq 1)$. To what extent one can assume the Gysin map:

$$
H_{m+1}(G ; \mathbb{Z}) \stackrel{\cap u}{\longrightarrow} H_{m-1}(W ; \mathbb{Z})
$$

to be injective (here $u \in H^{2}(G, G-W ; \mathbb{Z})$ denotes the orientation class [8], §19.2)?

Of course the answer to such a question is trivially affirmative in many cases. If Tor $H_{m+1}(G ; \mathbb{Z})=0$ or if we would work with $\mathbb{Q}$-coefficients then the Gysin map is injective by Hard Lefschetz Theorem. If $W$ is smooth then the Gysin map is injective by Lefschetz Hyperplane Theorem. However, it is easy to find examples where the above Gysin map is not injective, see Example 2.2. Unfortunately, in our case $W$ could be singular. The only way to obtain an interesting result is to vary $W$. If the linear system $|W|$ was very ample outside its base locus, then we could deduce the injectivity of (1) from Lefschetz Theorem with Singularities, see ([10], p. 199), and compare with (4], Lemma 3.2). Unfortunately, in our case $|W|$ may not be very ample outside its base locus. This is the ultimate reason for which the following Theorem, which is the main technical result of this paper, has required a major effort.

Theorem 1.3. Keep notations as in Theorem 1.1, set $G:=G_{i}, m=2 r:=\operatorname{dim}_{\mathbb{C}} G$, and define $W:=G \cap X\left(X \in\left|\mathcal{V}_{d}\right|\right.$ is Zariski general containing $\left.Z\right)$. Then the Gysin map

$$
k_{\star}: H_{m+1}(G ; \mathbb{Z}) \longrightarrow H_{m-1}(W ; \mathbb{Z})
$$

is injective.

Remark 1.4. The following example shows that the condition $d_{i} \neq d_{j}$ in Theorem 1.1 is necessary. Consider $Y=\mathbb{P}^{5}$. Let $G_{1}$ be a smooth quadric hypersurface, and let $L_{1}$ be a general hyperplane section of $G_{1}$. Let $G_{2}$ be a smooth general quadric hypersurface containing $L_{1}$, so that $G_{1} \cap G_{2}$ is equal to the union of $L_{1}$ with another smooth quadric threefold $L_{2}$. Let $X$ be a general hypersurface of 
degree $d>2$, and define $\Delta:=Z:=X \cap G_{1} \cap G_{2}$. Then $\Delta$ has two irreducible components $\Delta=Z_{1} \cup Z_{2}$, with $Z_{i}=X \cap L_{i}$. Now in $H^{4}(X ; \mathbb{Z})$ we have $Z_{1}=2 H^{2}$, where $H$ denotes the hyperplane class. Therefore $H^{4}(X ; \mathbb{Z})_{\Delta}$ is generated by $H^{2}$, which contradicts Theorem 1.1. (1).

\section{Some BAsic FACTS}

Notations 2.1. (i) From now on, unless it is otherwise stated, all cohomology and homology groups are with $\mathbb{Z}$-coefficients.

(ii) Borel-Moore homology. We will denote by $H_{i}^{B M}(M)$ the Borel-Moore homology groups of a variety $M$. Here we recall some properties of these groups, which will be needed throughout the paper.

a) Borel-Moore homology is equal to ordinary homology for any compact variety ([9], p. 217, line 7 from below).

b) If $U$ is open in $M$, and $C$ is the complement of $U$ in $M$, then there is a long exact sequence

$$
\cdots \rightarrow H_{i+1}^{B M}(U) \rightarrow H_{i}^{B M}(C) \rightarrow H_{i}^{B M}(M) \rightarrow H_{i}^{B M}(U) \rightarrow H_{i-1}^{B M}(C) \rightarrow \ldots
$$

([9], Lemma 3, p. 219).

c) If $M$ is smooth of complex dimension $m$, then there is a natural isomorphism

$$
H_{i}^{B M}(M) \cong H^{2 m-i}(M)
$$

([9], (26), p. 217).

Example 2.2. Denote by $T$ an irreducible, projective, smooth threefold such that Tor $H_{3}(T) \neq 0$. Choose a torsion class $0 \neq c \in$ Tor $H_{3}(T)$ and assume that $l \cdot c=0$ for some $l \in \mathbb{Z}$ with $l>0$. Define

$$
S:=T \times \mathbb{P}^{r-1} \subset G:=T \times \mathbb{P}^{m-3} \subset \mathbb{P}, \quad 2 r=m \geq 8,
$$

and choose a general $W \in\left|H^{0}\left(G, \mathcal{I}_{S, G}(k l)\right)\right|, k \gg 0$. From $\operatorname{dim} S=\operatorname{codim} S+4$ it follows that the hypersurface $W$ gives rise to a section of the normal bundle $\mathcal{N}_{S, G}(k l)$ which vanishes in dimension four. Therefore, we have $\operatorname{dim} \operatorname{Sing} W=4$. Consider the cycle

$$
\gamma:=c \otimes\left[\mathbb{P}^{r-1}\right] \in H_{m+1}(S),
$$

and let $\gamma^{\prime}$ be the image of $\gamma$ in $H_{m+1}(G)$, via push-forward. Notice that $\gamma^{\prime} \neq 0$. From the commutative diagram

$$
\begin{aligned}
& \gamma \quad \in H_{m+1}(S) \quad \longrightarrow \quad H_{m+1}(G) \\
& \downarrow \quad \downarrow \quad \downarrow \\
& \gamma \cap k l[H] \in H_{m-1}(S) \longrightarrow H_{m-1}(W),
\end{aligned}
$$

where $[H] \in H^{2}(S)$ denotes the hyperplane class, it follows that the image of $\gamma^{\prime}$ in $H_{m-1}(W)$ vanishes. Hence the map $H_{m+1}(G) \rightarrow H_{m-1}(W)$ provides an example of Gysin map, which is not injective. 
Remark 2.3. As we have just observed, in the examples above $\operatorname{dim} \operatorname{Sing} W=4$. We do not know examples of not injective Gysin maps for hypersurfaces with isolated singularities. Keeping notations as in Theorem 1.1, isolated singularities appear for instance when we define $W=G \cap \bar{X}, G=G_{i}$ ([7], Proposition 4.2.6 and proof, p. 133). Nevertheless, even in the case $\operatorname{dim} \operatorname{Sing} W=0$ it seems unlikely that Gysin map must be always injective. Indeed, assume $\operatorname{dim} \operatorname{Sing} W=0$ and define

$$
\Gamma:=\operatorname{Sing} W=\left\{x_{1}, \ldots, x_{s}\right\}, W^{\prime}:=W-\Gamma .
$$

Using (2) and (3) we have an isomorphism for $m>2$ :

$$
H_{m-1}(W) \cong H_{m-1}^{B M}\left(W^{\prime}\right) \cong H^{m-1}\left(W^{\prime}\right) .
$$

Consider the cohomology long exact sequence

$$
\ldots \longrightarrow H^{m-1}\left(W, W^{\prime}\right) \longrightarrow H^{m-1}(W) \longrightarrow H^{m-1}\left(W^{\prime}\right) \longrightarrow \ldots
$$

Choose a small ball $S_{j} \subset G$ around each $x_{j}$, and set $B_{j}:=S_{j} \cap W$ and $B_{j}^{0}:=$ $B_{j}-\left\{x_{j}\right\}$. By excision, we have

$$
H^{m-1}\left(W, W^{\prime}\right) \cong \bigoplus_{j=1}^{s} H^{m-1}\left(B_{j}, B_{j}^{0}\right)
$$

By $([5]$, p. 245), we have

$$
H^{m-1}\left(B_{j}, B_{j}^{0}\right) \cong H^{m-2}\left(K_{j}\right)
$$

where $K_{j}$ denotes the link of the singularity $x_{j}$. By Milnor's Theorem ([5], Theorem 3.2 .1 , p.76), the link is $(m-3)$-connected. Hence one cannot expect the last group vanishes. And in fact, when $m=2 r$ is even, for a node and more generally for an ordinary singularity one has $H^{m-2}\left(K_{j}\right) \neq 0$. Summing up, we have

$$
\begin{gathered}
\bigoplus_{j=1}^{s} H^{m-2}\left(K_{j}\right) \longrightarrow \\
\end{gathered}
$$

Although the vertical arrow is injective by Lefschetz Hyperplane Theorem, it seems unlikely that the oblique one, i.e. the Gysin map, must be injective for any $W$. However, we remark that for certain very special isolated singularities one knows that $H^{m-2}\left(K_{j}\right)=0([5]$, Proposition 4.7, p. 93, Theorem 4.10, p. 94). Finally, one can infer the injectivity of the Gysin map also when $\operatorname{rk} H^{m-2}(W)=\operatorname{rk} H_{m}(W)$. Indeed, in this case the exact sequence

$$
0 \rightarrow H^{m-2}(W) \rightarrow H_{m}(W) \rightarrow \bigoplus_{j=1}^{s} H^{m-2}\left(K_{j}\right) \rightarrow H^{m-1}(W) \rightarrow H_{m-1}(W)
$$

shows that the map $\bigoplus_{j=1}^{s} H^{m-2}\left(K_{j}\right) \rightarrow H^{m-1}(W)$ is injective, because $H^{m-2}\left(K_{j}\right)$ is torsion free $([5],(4.1)$ and (4.2), p. 91). By (44), this implies that the Gysin map $H_{m+1}(G) \rightarrow H_{m-1}(W)$ is injective, because its kernel is a torsion group by Hard Lefschetz Theorem. 
Notations 2.4. Consider a smooth quasi-projective variety $Y$ of dimension $n$ and a locally free sheaf $\mathcal{E}$ of rank $r$ on $Y$. Set $\mathbb{V}:=\mathbb{P}(\mathcal{E})$, denote by $\pi: \mathbb{V} \rightarrow Y$ the natural projection and denote by $c:=c_{1}\left(\mathcal{O}_{\mathbb{V}}(1)\right) \in A^{1}(\mathbb{V})$ the first Chern class. The cycle map ([8], p.370) sends $A^{i}(\mathbb{V})$ into the Borel-Moore homology group $H_{2(n+r-1-i)}^{B M}(\mathbb{V})$, which can be identified with $H^{2 i}(\mathbb{V})$, see (3). Denote by $\xi_{i} \in H^{2 i}(\mathbb{V})$ the cohomology class corresponding to $c^{i} \in A^{i}(\mathbb{V})$. By the LerayHirsch Theorem, we have an isomorphism for any fixed integer $m$ :

$$
\phi=\oplus_{i=0}^{r-1} \phi_{i}: \oplus_{i=0}^{r-1} H^{m-2 i}(Y) \rightarrow H^{m}(\mathbb{V}), \quad \phi_{i}(\cdot)=\pi^{*}(\cdot) \cup \xi_{i} .
$$

Now we are going to prove that the Leray-Hirsch Theorem holds true also for Borel-Moore homology groups. The following Lemma is certainly well-known, but we briefly prove it for lack of a suitable reference.

Lemma 2.5. We have an isomorphism of Borel-Moore homology groups:

$$
\psi=\oplus_{i=0}^{r-1} \psi_{i}: H_{m}^{B M}(\mathbb{V}) \rightarrow \oplus_{i=0}^{r-1} H_{m-2 i}^{B M}(Y), \quad \psi_{i}(\cdot)=\pi_{*}\left(\cdot \cap \xi_{i}\right) .
$$

Proof. As explained in ([15], Proof of the Leray-Hirsch Theorem, p. 195), we have an isomorphism in the derived category $D^{*}\left(A_{Y}\right)$, notations as in 6 ]

$$
\pi_{*} \mathbb{Z}_{\mathbb{V}} \cong \bigoplus_{i=0}^{r-1} \mathbb{Z}_{Y}[-2 i]
$$

In order to prove the Lemma it suffices to apply the derived functor $R^{\bullet} \Gamma_{c}$ to the isomorphism above and then take the dual:

$$
R^{\bullet} \Gamma_{c}(\mathbb{V}, \mathbb{Z}) \cong \bigoplus_{i=0}^{r-1} R^{\bullet} \Gamma_{c}(Y, \mathbb{Z})[-2 i], \quad D R^{\bullet} \Gamma_{c}(\mathbb{V}, \mathbb{Z}) \cong \bigoplus_{i=0}^{r-1} D R^{\bullet} \Gamma_{c}(Y, \mathbb{Z})[2 i]
$$

Compare with ([12, p.374), and use notations as in ([12], pp. 374-78).

Remark 2.6. (i) In the statement of the Leray-Hirsch Theorem the cohomology classes $\xi_{i}$ are defined up to classes in $\pi^{*}\left(H^{2 i}(Y)\right)$, hence $\xi_{r-1}$ could be replaced by the cycle class of any unisecant in $A_{n}(\mathbb{V})$.

(ii) Notice that $\pi$ is a local complete intersection (l.c.i. for short) morphism [8]. Set $M_{m}:=\operatorname{ker}\left(\oplus_{i=0}^{r-2} \psi_{i}\right)$. Then $\psi_{r-1}: M_{m} \rightarrow H_{m-2 r+2}^{B M}(Y)$ is an isomorphism with inverse the Gysin map

$$
\pi_{\star}: H_{m-2 r+2}^{B M}(Y) \rightarrow M_{m} \subset H_{m}^{B M}(\mathbb{V}),
$$

which represents the tensor product with the fundamental class of the fiber of $\pi: \mathbb{V} \rightarrow Y$. Compare with ([] $]$, Example 19.2.1, p. 382), and with the proof of Theorem 8 in ([14], Theorem 8, p. 258).

Notations 2.7. Choose a section in $H^{0}(Y, \mathcal{E})$, and assume it vanishes on a subscheme $D \subset Y$ having the right codimension. Then we have a surjection

$$
\mathcal{E}^{\vee} \longrightarrow \mathcal{I}_{D, Y} \longrightarrow 0
$$


This surjection induces an imbedding $\tilde{Y}:=B l_{D}(Y) \subset \mathbb{V}$. Since the natural projection $\pi^{Y}:=\left.\pi\right|_{\tilde{Y}}: \tilde{Y} \longrightarrow Y$ is a l.c.i. morphism of codimension 0, it follows that there exists a Gysin map ([8], Example 19.2.1, p. 382):

$$
\jmath_{\star}: H_{\bullet}^{B M}(Y) \rightarrow H_{\bullet}^{B M}(\tilde{Y}) .
$$

Theorem 2.8. With notations as above we have:

$$
\pi_{*}^{Y} \circ J_{\star}=\mathrm{id}: H_{\bullet}^{B M}(Y) \rightarrow H_{\bullet}^{B M}(Y),
$$

in particular $\jmath_{\star}$ is injective.

Proof. We denote by $f: \tilde{Y} \rightarrow \mathbb{V}$ the inclusion morphism. Applying (5) to the definition of Gysin map ([8], Example 19.2.1, p. 382) we have:

$$
\jmath_{\star}(x)=\pi_{\star}(x) \cap u_{\widetilde{Y}}, \quad \forall x \in H_{m}^{B M}(Y) .
$$

Here $u_{\tilde{Y}}$ denotes the orientation class of $\tilde{Y}$ in $\mathbb{V}([8]$, p. 372), so that:

$$
\cap u_{\tilde{Y}}: H_{\bullet}^{B M}(\mathbb{V}) \longrightarrow H_{\bullet-2 r+2}^{B M}(\tilde{Y}) .
$$

Since $\tilde{Y}$ is unisecant in $\mathbb{V}$, Remark 2.6. $(i)$, implies that we may assume $\xi_{r-1}$ to be the cycle class of $\tilde{Y}$. We thus get

$$
f_{*}\left(\cdot \cap u_{\widetilde{Y}}\right)=\cdot \cap \xi_{r-1} .
$$

According to (6), we have

$$
f_{*}\left(J_{\star}(x)\right)=f_{*}\left(\pi_{\star}(x) \cap u_{\tilde{Y}}\right)=\pi_{\star}(x) \cap \xi_{r-1}, \quad \forall x \in H_{m}^{B M}(Y) .
$$

Using (7), Lemma 2.5 and Remark 2.6, (ii), we may conclude

$$
\left(\pi_{*}^{Y} \circ \jmath_{\star}\right)(x)=\pi_{*}\left(f_{*}\left(\jmath_{\star}(x)\right)\right)=\pi_{*}\left(\pi_{\star}(x) \cap \xi_{r-1}\right)=\psi_{r-1} \circ \pi_{\star}(x)=x,
$$

for any $x \in H_{\bullet}^{B M}(Y)$.

Remark 2.9. Consider a quasi-projective smooth variety $Y$ and a complete intersection $\Delta=\bigcap_{i=1}^{r} X_{i}, X_{i} \in\left|H^{0}\left(Y, \mathcal{O}_{Y}\left(d_{i}\right)\right)\right|$. Fix $i_{0}$, set $X:=X_{i_{0}}$, and assume that $X$ is smooth. Applying Theorem 2.8 to $Y$ and $\mathcal{E}=\oplus_{i=1}^{r} \mathcal{O}_{Y}\left(d_{i}\right)$, and to $X$ and $\mathcal{E}=\oplus_{i=1, i \neq i_{0}}^{r} \mathcal{O}_{X}\left(d_{i}\right)$, we see that the Gysin maps are injective:

$$
\begin{gathered}
\jmath_{\star}: H_{\bullet}^{B M}(Y) \hookrightarrow H_{\bullet}^{B M}(\tilde{Y}), \quad \tilde{Y}:=B l_{\Delta}(Y), \\
\iota_{\star}: H_{\bullet}^{B M}(X) \hookrightarrow H_{\bullet}^{B M}(\tilde{X}), \quad \tilde{X}:=B l_{\Delta}(X) .
\end{gathered}
$$

Notice that $\tilde{X} \subset \widetilde{Y}([8]$, B.6.9, p.436), and that $\widetilde{X}$ is a Cartier divisor on $\tilde{Y}$, for $\Delta$ is regularly imbedded in both $X$ and $Y$ ([8], B.6.10, p.437).

Lemma 2.10. Denote by $\iota^{X}: X \rightarrow Y$ and $\iota^{\tilde{X}}: \widetilde{X} \rightarrow \widetilde{Y}$ the inclusions. Then the following diagram of Gysin maps is commutative:

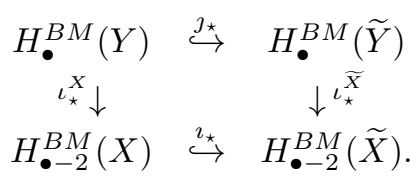


Proof. The natural maps $\tilde{X} \stackrel{\pi^{X}}{\longrightarrow} X \stackrel{\iota^{X}}{\longrightarrow} Y$ and $\widetilde{X} \stackrel{\iota^{\widetilde{X}}}{\longrightarrow} \widetilde{Y} \stackrel{\pi^{Y}}{\longrightarrow} Y$ are equal. Furthermore, they are l.c.i. maps because they are both composite of l.c.i. maps. Therefore, by functoriality of the Gysin morphism ([8], Example 19.2.1, p. 382), we have:

$$
\imath_{\star} \circ \iota_{\star}^{X}=\iota_{\star}^{\widetilde{X}} \circ J_{\star}
$$

Notations 2.11. Let $Y \subset \mathbb{P}$ be a possibly singular quasi-projective variety, and set $\mathcal{V}_{d}:=\operatorname{Im}\left(H^{0}\left(\mathbb{P}, \mathcal{O}_{\mathbb{P}}(d)\right) \rightarrow H^{0}\left(Y, \mathcal{O}_{Y}(d)\right)\right)$. Consider a complete intersection $\Delta=\bigcap_{1}^{r} X_{i}, X_{i} \in\left|\mathcal{V}_{d_{i}}\right|$, with $d:=d_{1} \geq d_{2} \geq d_{3} \geq \cdots \geq d_{r}$. Fix a hypersurface $X \in\left|\mathcal{V}_{\Delta, d}\right|$, where $\mathcal{V}_{\Delta, d}:=\mathcal{V}_{d} \cap H^{0}\left(Y, \mathcal{I}_{\Delta, Y}(d)\right)$. Then we have

$$
\widetilde{X}:=B l_{\Delta}(X) \subset B l_{\Delta}(Y)=: \tilde{Y}
$$

(8], B.6.9, p.436). Since $\Delta$ is regularly imbedded in both $X$ and $Y$, it follows that $\widetilde{X}$ is a Cartier divisor on $\widetilde{Y}\left(\left[8\right.\right.$, B.6.10, p.437). More precisely $\widetilde{X} \in\left|H^{0}\left(\mathcal{O}_{\widetilde{Y}}(d \widetilde{H}-\widetilde{\Delta})\right)\right|$, where $\mathcal{O}_{\widetilde{Y}}(\widetilde{H})$ denotes the pull-back of $\mathcal{O}_{Y}(1)$ via the natural projection $\tilde{Y} \rightarrow Y$, and $\widetilde{\Delta}$ denotes the exceptional divisor in $\tilde{Y}$. Since $\mathcal{I}_{\Delta, Y}(d)$ is globally generated, by letting $X \in\left|\mathcal{V}_{\Delta, d}\right|$ vary, we have a base point free linear system $|\widetilde{X}|$ on $\tilde{Y}$ and a morphism

$$
\nu: \tilde{Y} \rightarrow \mathbb{P}^{\prime}=\mathbb{P}\left(\mathcal{V}_{\Delta, d}^{*}\right), \quad \mathcal{Q}:=\nu(\tilde{Y}) .
$$

Proposition 2.12. Assume moreover that $d>d_{2}$ and set $\mathcal{T}:=\bigcap_{i=2}^{r} X_{i}$. Then we have:

(1) $\mathcal{T} \cong \tilde{\mathcal{T}}:=B l_{\Delta}(\mathcal{T}) \subset \tilde{Y}, \tilde{\mathcal{T}} \cap \tilde{X}=\emptyset$, hence the morphism $\nu$ sends $\tilde{\mathcal{T}}$ to a point $p \in \mathcal{Q}$;

(2) the morphism $\nu$ is an isomorphism outside $\widetilde{\mathcal{T}}$, namely $|\widetilde{X}|$ is very ample on $\tilde{Y}-\tilde{\mathcal{T}}$ :

$$
\nu: \widetilde{Y}-\widetilde{\mathcal{T}} \cong \mathcal{Q}-\{p\} .
$$

Proof. (1) Since $\Delta$ is a Cartier divisor cut out on $\mathcal{T}$ by $X$, it follows that the natural projection

$$
\pi: \widetilde{\mathcal{T}} \rightarrow \mathcal{T}
$$

is in fact an isomorphism. So we have $\mathcal{T} \cong \widetilde{\mathcal{T}}=B l_{\Delta}(\mathcal{T}) \subset \widetilde{Y}$. Furthermore, we have:

$$
\mathcal{O}_{\widetilde{Y}}(-\widetilde{\Delta}) \otimes \mathcal{O}_{\widetilde{\mathcal{T}}} \cong \pi^{*}\left(\mathcal{O}_{\mathcal{T}}(-\Delta)\right) \cong \pi^{*}\left(\mathcal{I}_{X \cap \mathcal{T}, \mathcal{T}}\right) \cong \mathcal{O}_{\widetilde{Y}}(-\widetilde{\Delta}-\widetilde{X}) \otimes \mathcal{O}_{\widetilde{\mathcal{T}}}
$$

Hence we find

$$
\mathcal{O}_{\widetilde{Y}}(\widetilde{X}) \otimes \mathcal{O}_{\widetilde{\mathcal{T}}} \cong \mathcal{O}_{\widetilde{\mathcal{T}}}
$$

and we are done.

(2) Consider the point $p \in \mathbb{P}^{\prime}$ representing the hyperplane $L \subset\left|\mathcal{V}_{\Delta, d}\right|$ spanned by divisors of the form $X_{i} \cup M_{i}$, with $i \geq 2$ and $M_{i} \in\left|\mathcal{V}_{d-d_{i}}\right|$. Such a hyperplane is spanned by the image of $\left(\mathcal{V}_{d_{2}} \cap H^{0}\left(Y, \mathcal{I}_{\mathcal{T}, Y}\left(d_{2}\right)\right) \otimes \mathcal{V}_{d-d_{2}}\right.$ in $\left|\mathcal{V}_{\Delta, d}\right|$. Since its base locus is $\mathcal{T}$, it follows that $\nu(\widetilde{\mathcal{T}})=p$. On the other hand ([8], B.6.10 p.437), we have:

$$
N_{\widetilde{\mathcal{T}}, \widetilde{Y}} \cong\left(\pi^{*} N_{\mathcal{T}, Y}\right)(-\widetilde{\Delta}) \cong \oplus_{i=2}^{r-1} \mathcal{O}_{\widetilde{Y}}\left(\widetilde{X}_{i}\right)
$$


It follows that $\tilde{\mathcal{T}}$ is a complete intersection also in $\tilde{Y}$ :

$$
\bigcap_{i=2}^{r-1} \tilde{X}_{i}=\widetilde{\mathcal{T}} \subset \tilde{Y}
$$

But the hyperplane $L \subset \mathbb{P}^{\prime} \cong|\widetilde{X}|^{*}$ is spanned by divisors of the form $\widetilde{X}_{i} \cup \widetilde{M}_{i}$, with $i \geq 2, M_{i} \in\left|\mathcal{V}_{d-d_{i}}\right|$, and $\widetilde{M}_{i}:=$ strict transform of $M_{i}$ in $\tilde{Y}$. Since the base locus of $L$ is $\tilde{\mathcal{T}}$, it follows that $\nu^{-1}(p)=\widetilde{\mathcal{T}}$ scheme theoretically. Consider a point $x \in \widetilde{Y}-\widetilde{\mathcal{T}}$ and its image $\nu(x) \neq p$. The corresponding hyperplane $L_{x} \neq L \subset \mathcal{V}_{\Delta, d}$ must contain a divisor $X \in L_{x}$ such that $\Delta=X \cap \mathcal{T}$. If $\nu$ did not separate $x$ from another point or a tangent vector, then they both would be contained in $\widetilde{X}:=B l_{\Delta}(X)$. This is impossible because $\mathcal{I}_{\Delta, X}\left(d_{2}\right)$ is generated by $\mathcal{V}_{\Delta, d_{2}}$, hence our linear system is very ample on $\tilde{X}$ (recall that $d>d_{2}$ ).

\section{Proof of Theorem 1.3}

Notations 3.1. Let $Y$ be a smooth projective variety of dimension $m=2 r+1$, and let $\bar{X} \in\left|\mathcal{V}_{d}\right|, G_{i} \in\left|\mathcal{V}_{d_{i}}\right|, 1 \leq i \leq r$, be a regular sequence of smooth hypersurfaces. Assume moreover that $d>d_{1}>\cdots>d_{r}$. Define $\mathcal{T}:=\bigcap_{i=1}^{r} G_{i}$ and $\Delta:=\mathcal{T} \cap \bar{X}$ and fix $G=G_{i_{0}}$. If $X \in\left|\mathcal{V}_{\Delta, d}\right|$ denotes a general hypersurface containing $\Delta$, define also

$$
W:=X \cap G
$$

Consider the Gysin map

$$
k_{\star}: H_{\bullet}(G) \longrightarrow H_{\bullet-2}(W),
$$

where $k: W \rightarrow G$ denotes the imbedding morphism.

Theorem 1.3 will follow from a slightly stronger result:

Theorem 3.2. The Gysin map

$$
k_{\star}: H_{m+1}(G) \longrightarrow H_{m-1}(W)
$$

is injective for a general $W \in\left|\mathcal{V}_{d} \cap H^{0}\left(G, \mathcal{I}_{\Delta, G}(d)\right)\right|$.

We start with:

Proposition 3.3. Assume $r \geq 2$ and define $\mathcal{T}:=\bigcap_{i=1}^{r} G_{i}$. Assume $x \in H_{m+1}(G)$ is such that $k_{\star}(x)=0 \in H_{m-1}(W)$, for a general $W \in\left|\mathcal{V}_{d} \cap H^{0}\left(G, \mathcal{I}_{\Delta, G}(d)\right)\right|$. Then $x$ belongs to the image of the push forward from $\mathcal{T}$ :

$$
x \in \operatorname{Im}\left(h_{*}: H_{m+1}(\mathcal{T}) \rightarrow H_{m+1}(G)\right) .
$$

Proof. Denote by $S:=\operatorname{Sing} \Delta$ the singular locus of $\Delta$, and set

$$
\Delta^{0}:=\Delta-S, \quad \mathcal{T}^{0}:=\mathcal{T}-S, G^{0}:=G-S, \quad W^{0}:=W-S .
$$

Observe that $\Delta^{0}, G^{0}$ and $W^{0}$ are smooth. Since $\operatorname{dim} S \leq r-1$ ([3], Proof of Theorem 1.2), it follows that $H_{m+1}(S)=H_{m}(S)=0$ ([9], Lemma 4, p. 219). Therefore, from the exact sequence for Borel-Moore homology:

$$
\ldots \longrightarrow H_{m+1}(S) \longrightarrow H_{m+1}(G) \longrightarrow H_{m+1}^{B M}\left(G^{0}\right) \longrightarrow H_{m}(S) \longrightarrow \ldots
$$


we get $H_{m+1}(G) \cong H_{m+1}^{B M}\left(G^{0}\right)$ (compare with (2) $)$. We thus find $x \in H_{m+1}^{B M}\left(G^{0}\right)$, and therefore $k_{\star}(x)=0 \in H_{m-1}^{B M}\left(W^{0}\right)$.

Combining Theorem 2.8 and Lemma 2.10 we have moreover a commutative diagram with injective horizontal maps:

$$
\begin{array}{ccc}
H_{m+1}^{B M}\left(G^{0}\right) & \stackrel{\jmath_{\star}^{\star}}{\hookrightarrow} & H_{m+1}^{B M}\left(\widetilde{G}^{0}\right) \\
k_{\star} \downarrow & & \tilde{k}_{\star} \downarrow \\
H_{m-1}^{B M}\left(W^{0}\right) & \stackrel{\iota_{\star}}{\hookrightarrow} & H_{m-1}^{B M}\left(\widetilde{W}^{0}\right),
\end{array}
$$

with $\widetilde{G^{o}}:=B l_{\Delta^{0}}\left(G^{0}\right)$ and $\widetilde{W^{0}}:=B l_{\Delta^{0}}\left(W^{0}\right)$. We thus find

$$
\tilde{x}:=\jmath_{\star}(x) \in H_{m+1}^{B M}\left(\widetilde{G^{o}}\right) \text {, with } \tilde{k}_{\star}(\tilde{x})=0 \in H_{m-1}^{B M}\left(\widetilde{W}^{0}\right) .
$$

Let us look at the exact sequence:

$$
\ldots \longrightarrow H_{m+1}^{B M}\left(\widetilde{\mathcal{T}}^{0}\right) \stackrel{\sigma}{\longrightarrow} H_{m+1}^{B M}\left(\widetilde{G}^{0}\right) \stackrel{\rho}{\longrightarrow} H_{m+1}^{B M}\left(\widetilde{G}^{0}-\widetilde{\mathcal{T}}^{0}\right) \longrightarrow \ldots
$$

$\left(\mathcal{T}^{0} \cong \widetilde{\mathcal{T}}^{0} \cong B l_{\Delta^{0}}\left(\mathcal{T}^{0}\right)\right)$. Applying Notations 2.11 and Proposition 2.12 to the linear system $\left|\widetilde{W}^{0}\right|$ on $\widetilde{G}^{0}$, we find that $\widetilde{W}^{0} \cap \widetilde{\mathcal{T}}^{0}=\emptyset$. Then the linear system $\widetilde{W}^{0}$ is very ample on the smooth variety $\widetilde{G}^{0}-\widetilde{\mathcal{T}}^{0}$. Since

$$
\tilde{k}_{\star}(\tilde{x})=0 \in H_{m-1}^{B M}\left(\widetilde{W}^{0}\right) \cong H^{m-1}\left(\widetilde{W}^{0}\right),
$$

it follows by Lefschetz Theorem with Singularities ([10], p.199) that:

$$
\rho(\tilde{x})=0 \in H_{m+1}^{B M}\left(\widetilde{G}^{0}-\widetilde{\mathcal{T}}^{0}\right) \cong H^{m-1}\left(\widetilde{G}^{0}-\widetilde{\mathcal{T}}^{0}\right) .
$$

Then (8) implies $\tilde{x}=\sigma(y) \in \operatorname{Im}\left(H_{m+1}^{B M}\left(\widetilde{\mathcal{T}}^{0}\right) \rightarrow H_{m+1}^{B M}\left(\widetilde{G}^{0}\right)\right)$. We are done because $y \in H_{m+1}^{B M}\left(\widetilde{\mathcal{T}}^{0}\right) \cong H_{m+1}^{B M}\left(\mathcal{T}^{0}\right) \cong H_{m+1}(\mathcal{T})$, and $h_{*}(y) \in H_{m+1}(G)$ must coincide with $x$. In fact they both go to $\tilde{x} \in H_{m+1}^{B M}\left(\widetilde{G}^{0}\right)([9]$, p. 219, Exercise 5), and the map

$$
H_{m+1}(G) \cong H_{m+1}^{B M}\left(G^{0}\right) \rightarrow H_{m+1}^{B M}\left(\widetilde{G}^{0}\right)
$$

is injective by Theorem 2.8 ,

Proposition 3.4. Assume $r \geq 2$ and define $\mathcal{T}:=\bigcap_{i=1}^{r} G_{i}$. If $y \in H_{m+1}(\mathcal{T})$ is such that $h_{*}(y) \in \operatorname{Tor}\left(H_{m+1}(G)\right)$ then $y=0$.

Proof. First notice that $\operatorname{Tor}\left(H_{m+1}(\mathcal{T})\right)=0$. In fact, since $\operatorname{dim} \operatorname{Sing} \mathcal{T} \leq r-2([3]$, Proof of Theorem 1.2), it follows that

$$
H_{m+1}(\mathcal{T}) \cong H_{m+1}^{B M}(\mathcal{T}-\operatorname{Sing} \mathcal{T}) \cong H^{1}(\mathcal{T}-\operatorname{Sing} \mathcal{T})
$$

Furthermore, $H^{1}(\mathcal{T}-\operatorname{Sing} \mathcal{T})$ is torsion free by the Universal Coefficient Theorem ([14], p. 243). From Tor $\left(H_{m+1}(\mathcal{T})\right)=0$ it follows $H_{m+1}(\mathcal{T} ; \mathbb{Z}) \subset H_{m+1}(\mathcal{T} ; \mathbb{Q})$, and we may assume $y \in H_{m+1}(\mathcal{T} ; \mathbb{Q})$ is such that $0=h_{*}(x) \in H_{m+1}(G ; \mathbb{Q})$. From now on, in the rest of the proof, all cohomology and homology groups are with $\mathbb{Q}$-coefficients.

We are going to argue by induction on $r \geq 2$.

- $r=2$.

In this case, by ([7, Proposition 4.2.6, p.133), we know that $\mathcal{T}=G_{1} \cap G_{2}$ is a threefold with isolated singularities (see also [3, loc. cit.). Set $\Gamma:=\operatorname{Sing} \mathcal{T}=$ 
$\left\{x_{1}, \ldots, x_{s}\right\}, \mathcal{T}^{\prime}:=\mathcal{T}-\Gamma$. Then $y \in H_{5}(\mathcal{T}) \cong H_{5}^{B M}\left(\mathcal{T}^{\prime}\right) \cong H^{1}\left(\mathcal{T}^{\prime}\right)$. We claim that:

$$
y \in \operatorname{Im}\left(H^{1}(\mathcal{T}) \rightarrow H^{1}\left(\mathcal{T}^{\prime}\right)\right)
$$

From the cohomology exact sequence:

$$
\ldots \longrightarrow H^{1}(\mathcal{T}) \longrightarrow H^{1}\left(\mathcal{T}^{\prime}\right) \longrightarrow H^{2}\left(\mathcal{T}, \mathcal{T}^{\prime}\right) \longrightarrow \ldots
$$

we see that in order to prove the claim it suffices to show that $H^{2}\left(\mathcal{T}, \mathcal{T}^{\prime}\right)=0$. Choose a small ball $S_{j} \subset G$ around each $x_{j}$, and set $B_{j}:=S_{j} \cap W$ and $B_{j}^{0}:=$ $B_{j}-\left\{x_{j}\right\}$. Then by excision we have

$$
H^{2}\left(\mathcal{T}, \mathcal{T}^{\prime}\right) \cong \bigoplus_{j=1}^{s} H^{2}\left(B_{j}, B_{j}^{0}\right) \cong \bigoplus_{j=1}^{s} H^{1}\left(K_{j}\right),
$$

where $K_{j}$ denotes the link of the singularity $x_{j}$ ([5], p. 245). The claim (9) follows by Milnor's Theorem ([5], Theorem 3.2.1, p.76). To conclude the proof in the case $r=2$ it suffices to observe that any $y \in H^{1}(\mathcal{T}) \cong H^{1}(G)$ such that $0=h_{*}(y) \in$ $H_{5}(G) \cong H^{3}(G)$ vanishes by Hard Lefschetz Theorem. Recall that now we are assuming that all cohomology and homology groups are with $\mathbb{Q}$-coefficients.

- $r \geq 3$.

Set $R:=G \cap G_{j}, j \neq i_{0}$, and denote by $f: \mathcal{T} \rightarrow R$ the inclusion morphism. We claim that:

$$
z:=f_{*}(y)=0 \in H_{m+1}(R) .
$$

First we have

$$
\psi_{*}(z)=\psi_{*}\left(f_{*}(y)\right)=(\psi \circ f)_{*}(y)=h_{*}(y)=0 \in H_{m+1}(G),
$$

with $\psi: R \rightarrow G$ the inclusion morphism. By ([7], Proposition 4.2.6, p.133), $R$ has at worst finitely many singularities. Set

$$
\Gamma:=\operatorname{Sing} R=\left\{x_{1}, \ldots, x_{s}\right\}, \quad R^{\prime}:=R-\Gamma .
$$

Then $z \in H_{m+1}(R) \cong H_{m+1}^{B M}\left(R^{\prime}\right) \cong H^{m-3}\left(R^{\prime}\right)$. Consider the cohomology long exact sequence:

$$
\ldots \longrightarrow H^{m-3}(R) \longrightarrow H^{m-3}\left(R^{\prime}\right) \longrightarrow H^{m-2}\left(R, R^{\prime}\right) \longrightarrow \ldots,
$$

choose a small ball $S_{j} \subset G$ around each $x_{j}$, and set $B_{j}:=S_{j} \cap R$ and $B_{j}^{0}:=$ $B_{j}-\left\{x_{j}\right\}$. By excision we have

$$
H^{m-2}\left(R, R^{\prime}\right) \cong \bigoplus_{j=1}^{s} H^{m-2}\left(B_{j}, B_{j}^{0}\right),
$$

and by ([5], p. 245) we get:

$$
H^{m-2}\left(B_{j}, B_{j}^{0}\right) \cong \bigoplus_{j=1}^{s} H^{m-3}\left(K_{j}\right)=0 .
$$

Here $K_{j}$ denotes the link of the singularity $x_{j}$. The last vanishing follows by Milnor's Theorem ([5], Theorem 3.2.1, p.76), because the link of an isolated singularity of dimension $\operatorname{dim} R=m-1$ is $(m-3)$-connected. 
Combining (11), (12), (13) and (14) we have

$$
z \in H^{m-3}(R) \cong H^{m-3}(G), \quad 0=\psi_{*}(z) \in H_{m+1}(G) \cong H^{m-1}(G),
$$

and our claim (10) follows by Hard Lefschetz Theorem.

Having proved $f_{*}(y)=0$, we now recall that $\operatorname{dim} \operatorname{Sing} \mathcal{T} \leq r-2$ by ([3], Proof of Theorem 1.2). Then we can choose a general hyperplane $H$ and look at the following commutative diagram:

$$
\begin{aligned}
& H_{m+1}(\mathcal{T}) \cong H^{1}\left(\mathcal{T}^{\prime}\right) \quad \stackrel{f_{*}}{\longrightarrow} \quad H_{m+1}(R) \cong H^{m-3}\left(R^{\prime}\right) \\
& \downarrow \\
& H_{m-1}(\mathcal{T} \cap H) \cong H^{1}\left(\mathcal{T}^{\prime} \cap H\right) \quad \longrightarrow \quad H_{m-1}(R \cap H) \cong H^{m-3}\left(R^{\prime} \cap H\right),
\end{aligned}
$$

where $\mathcal{T}^{\prime}:=\mathcal{T}-\operatorname{Sing} \mathcal{T}$, and the vertical maps are injective by Lefschetz Theorem with Singularities ([10], p. 199). The statement follows by induction.

Proof of Theorem 3.2. Choose an element $0 \neq x \in H_{m+1}(G)$. We have to prove $0 \neq k_{\star}(x) \in H_{m-1}(W)$. We distinguish two cases, according that either $r=1$ or $r \geq 2$.

If $r=1$ then we may assume $x \in H_{3}(G ; \mathbb{Q})$ because Tor $H_{3}(G) \cong$ Tor $H^{1}(G)=0$ by the Universal Coefficient Theorem. And the claim follows because the composite of $k_{\star}$ with the push-forward (put $m=2$ ):

$$
H^{m-1}(G ; \mathbb{Q}) \cong H_{m+1}(G ; \mathbb{Q}) \stackrel{k_{\star}}{\longrightarrow} H_{m-1}(W ; \mathbb{Q}) \longrightarrow H_{m-1}(G ; \mathbb{Q}) \cong H^{m+1}(G ; \mathbb{Q})
$$

is injective by Hard Lefschetz Theorem.

Next assume $r \geq 2$. If $x \notin \operatorname{Tor}\left(H_{m+1}(G)\right)$ then again we may assume $x \in$ $H_{m+1}(G ; \mathbb{Q})$, and we may conclude as before. If $0 \neq x \in \operatorname{Tor}\left(H_{m+1}(G)\right)$ then we have $k_{\star}(x) \neq 0$ just combining Propositions 3.3 and 3.4 .

\section{Proof of Theorem 1.2}

Notations 4.1. Applying Proposition 2.12, and Notations 2.11, to the complete intersection $W=X \cap G$ of Theorem 3.2, we get a morphism

$$
\tilde{Y}:=B l_{W}(Y) \longrightarrow \mathcal{Q} \subset \mathbb{P}\left(\mathcal{V}_{W, d}^{*}\right) \quad \mathcal{V}_{W, d}:=\mathcal{V}_{d} \cap H^{0}\left(Y, \mathcal{I}_{W, Y}(d)\right)
$$

This map contracts $G \cong \widetilde{G}:=B l_{W}(G) \subset \widetilde{Y}$ to a point $p \in \mathcal{Q}$, and sends $\widetilde{Y}-\widetilde{G}$ isomorphically to $\mathcal{Q}-\{p\}$. By $([1]$, Remark 3.1), both $\widetilde{Y}$ and $\mathcal{Q}$ have at worst isolated singularities.

Corollary 4.2. The push-forward map:

$$
H_{m+2}(\tilde{Y}) \longrightarrow H_{m+2}(\mathcal{Q})
$$

is surjective, thus the cokernel of the map

$$
H_{m+2}(\tilde{Y}) \longrightarrow H^{m}(X)
$$

is torsion free, for a general $X \in\left|\mathcal{V}_{W, d}\right|$. 
Proof. From the commutative diagram

$$
\begin{array}{ccccccc}
H_{k}(\widetilde{G}) & \rightarrow & H_{k}(\tilde{Y}) & \rightarrow & H_{k}(\tilde{Y}, \widetilde{G}) & \rightarrow & H_{k-1}(\widetilde{G}) \\
\downarrow & & \downarrow & & \| & & \\
H_{k}(\{p\}) & \rightarrow & H_{k}(\mathcal{Q}) & \rightarrow & H_{k}(\mathcal{Q},\{p\}) & \rightarrow & H_{k-1}(\{p\})
\end{array}
$$

we see that $H_{m+2}(\tilde{Y}) \rightarrow H_{m+2}(\mathcal{Q})$ is surjective if the push-forward $H_{m+1}(\widetilde{G}) \rightarrow$ $H_{m+1}(\widetilde{Y})$ is injective, and this follows simply combining Theorem 3.2 with Corollary 2.6 of 4 . The last statement is direct consequence of the first. In fact

$$
\operatorname{coker}\left(H_{m+2}(\widetilde{Y}) \longrightarrow H^{m}(X)\right) \cong \operatorname{coker}\left(H_{m+2}(\mathcal{Q}) \longrightarrow H^{m}(X)\right),
$$

and the last group is torsion free by Lefschetz Theorem with Singularities ([10], p.199), because

$$
H_{m+2}(\mathcal{Q}) \cong H_{m+2}^{B M}(\mathcal{Q}-\operatorname{Sing} \mathcal{Q}) \cong H^{m}(\mathcal{Q}-\operatorname{Sing} \mathcal{Q}) .
$$

Remark 4.3. By Corollary 2.6 of $\left[4, H_{m+2}(\tilde{Y}) \cong H_{m+2}(Y) \oplus H_{m}(W)\right.$, hence Corollary 4.2 implies that the group

$$
\operatorname{coker}\left(H_{m+2}(Y) \oplus H_{m}(W) \rightarrow H^{m}(X)\right)
$$

has no torsion. In the morphism above the first component is intended to be the Gysin map followed by Poincaré duality, and the second one is intended to be the push forward followed by Poincaré duality.

Notations 4.4. Let $Y \subset \mathbb{P}$ be a smooth projective variety of dimension $m+1=$ $2 r+1 \geq 3$. Let $\bar{X}, G_{1}, \ldots, G_{r}$ be a regular sequence of smooth divisors in $Y$, with $\bar{X} \in\left|\mathcal{V}_{d}\right|$, each $G_{i} \in\left|\mathcal{V}_{d_{i}}\right|$, and such that $d>d_{1}>\cdots>d_{r}$. Set $\Delta:=\bar{X} \cap G_{1} \cap$ $\cdots \cap G_{r}$, and $W:=\bar{X} \cap G_{1}$. For any $1 \leq l \leq r-1$ fix general divisor $H_{l} \in\left|\mathcal{V}_{\mu_{l}}\right|$, with $0 \ll \mu_{1} \ll \cdots \ll \mu_{r-1}$, and for any $0 \leq l \leq r-1$ define $\left(Y_{l}, X_{l}, W_{l}, \Delta_{l}\right)$ as follows. For $l=0$ define $\left(Y_{0}, X_{0}, W_{0}, \Delta_{0}\right):=(Y, X, W, \Delta), X \in\left|\mathcal{V}_{W, d}\right|$ general. For $1 \leq l \leq r-1$ define $Y_{l}:=G_{1} \cap \cdots \cap G_{l} \cap H_{1} \cap \cdots \cap H_{l}, X_{l}:=X \cap Y_{l}$, $W_{l}:=X \cap Y_{l} \cap G_{l+1}$, and $\Delta_{l}:=\Delta \cap Y_{l}\left(m_{l}:=\operatorname{dim} X_{l}=m-2 l\right)$. Notice that $\operatorname{dim} Y_{r-1}=3$ and that $\Delta_{r-1}=W_{r-1}$.

Remark 4.5. (1) As in Theorem 1.2, define:

$$
\mathcal{V}_{l, d}:=\operatorname{Im}\left(H^{0}\left(\mathbb{P}, \mathcal{O}_{\mathbb{P}}(d)\right) \rightarrow H^{0}\left(Y_{l}, \mathcal{O}_{Y_{l}}(d)\right)\right) .
$$

(2) As in Notations 4.1 define:

$$
\tilde{Y}_{l}:=B l_{W_{l}}\left(Y_{l}\right) \longrightarrow \mathcal{Q}_{l} \subset \mathbb{P}\left(\mathcal{V}_{W_{l}, d}^{*}\right), \quad \mathcal{V}_{W_{l}, d}:=\mathcal{V}_{l, d} \cap H^{0}\left(Y_{l}, \mathcal{I}_{W_{l}, Y_{l}}(d)\right) .
$$

By Corollary 4.2 and Remark 4.3 the group

$$
\begin{gathered}
\mathbb{V}_{l}:=\operatorname{coker}\left(H_{m_{l}+2}\left(\mathcal{Q}_{l}\right) \rightarrow H^{m_{l}}\left(X_{l}\right)\right)= \\
=\operatorname{coker}\left(H_{m_{l}+2}\left(Y_{l}\right) \oplus H_{m_{l}}\left(W_{l}\right) \rightarrow H^{m_{l}}\left(X_{l}\right)\right)
\end{gathered}
$$

is torsion free. 
(3) By $\left(\left[2\right.\right.$, Theorem 1.1), $\mathbb{V}_{l} \otimes \mathbb{Q}$ supports an irreducible action of the monodromy group of the linear system $\left|\mathcal{V}_{W_{l}, d}\right|_{\left.\right|_{Y_{l}}}$. Moreover, by previous remark, we have $\mathbb{V}_{l} \subset \mathbb{V}_{l} \otimes \mathbb{Q}$.

Theorem 1.2 will follow from a slightly stronger result:

Theorem 4.6. Let $Y \subset \mathbb{P}$ be a smooth projective variety of dimension $m+1=$ $2 r+1 \geq 3$. Let $\bar{X}, G_{1}, \ldots, G_{r}$ be a regular sequence of smooth divisors in $Y$, with $\bar{X} \in\left|\mathcal{V}_{d}\right|$, each $G_{i} \in\left|\mathcal{V}_{d_{i}}\right|$, and such that $d>d_{1}>\cdots>d_{r}$. Set $\Delta:=$ $\bar{X} \cap G_{1} \cap \cdots \cap G_{r}$ and let $X \in\left|\mathcal{V}_{d} \cap H^{0}\left(Y, \mathcal{I}_{\Delta, Y}(d)\right)\right|$ be a very general hypersurface containing $\Delta$. Assume that the vanishing cohomology of $X$ is not of pure Hodge type $\left(\frac{m}{2}, \frac{m}{2}\right)$, denote by $H^{m}(X ; \mathbb{Z})_{\Delta}$ the subgroup of $H^{m}(X ; \mathbb{Z})$ generated by the components of $\Delta$ and by $H^{m}(X ; \mathbb{Z})_{\Delta^{-}}$the subgroup of $H^{m}(X ; \mathbb{Z})$ generated by the components of $\Delta$ except one. Then we have:

(1) $H^{m}(X ; \mathbb{Z})_{\Delta}$ is freely generated by the components of $\Delta ;$

(2) $N S_{r}(X ; \mathbb{Z})=\left[N S_{r}(X ; \mathbb{Z}) \cap H^{m}(Y ; \mathbb{Z})\right] \oplus H^{m}(X ; \mathbb{Z})_{\Delta^{-}}$;

(3) $N S_{r}(X ; \mathbb{Q})=N S_{r+1}(Y ; \mathbb{Q}) \oplus H^{m}(X ; \mathbb{Q})_{\Delta^{-}}$.

Proof of Theorem 4.6. The argument is very similar to that already used in the proof of Theorem 3.3 of [4], so we are going to be rather sketchy. Thanks to Theorem 1.2 of [4], it suffices to show that the cokernel of the map

$$
H_{m+2}(Y) \oplus H_{m}(\Delta) \longrightarrow H^{m}(X)
$$

is free. In order to prove this, we argue by decreasing induction on $l$ and prove that

$$
\mathbb{W}_{l}:=\operatorname{coker}\left(H_{m_{l}+2}\left(Y_{l}\right) \oplus H_{m_{l}}\left(\Delta_{l}\right) \longrightarrow H^{m_{l}}\left(X_{l}\right)\right)
$$

coincides with the group $\mathbb{V}_{l}$ defined in Remark 4.5. (2). For $l=r-1$ this is clear because $\Delta_{r-1}=W_{r-1}$, compare with Notations 4.4. Observe that we only need to prove the following inclusion:

$\operatorname{Im}\left(H_{m_{l}+2}\left(Y_{l}\right) \oplus H_{m_{l}}\left(\Delta_{l}\right) \longrightarrow H^{m_{l}}\left(X_{l}\right)\right) \supseteq \operatorname{Im}\left(H_{m_{l}+2}\left(Y_{l}\right) \oplus H_{m_{l}}\left(W_{l}\right) \longrightarrow H^{m_{l}}\left(X_{l}\right)\right)$

for the reverse inclusion is obvious. Notice that the composite:

$$
H_{m_{l}}\left(W_{l}\right) \hookrightarrow H_{m_{l+1}}\left(X_{l+1}\right) \longrightarrow \mathbb{W}_{l+1}
$$

vanishes because $\mathbb{W}_{l+1}=\mathbb{V}_{l+1}$ by induction, the monodromy acts irreducibly on $\mathbb{V}_{l+1}$ and we can assume that $\mathrm{rk} H_{m_{l}}\left(W_{l}\right) \ll \mathrm{rk} \mathbb{V}_{l+1}$. Again by induction we find that

$$
\operatorname{Im}\left(H_{m_{l}}\left(W_{l}\right) \hookrightarrow H_{m_{l+1}}\left(X_{l+1}\right)\right)
$$

is contained in

$$
\operatorname{Im}\left(H_{m_{l+1}+2}\left(Y_{l+1}\right) \oplus H_{m_{l+1}}\left(\Delta_{l+1}\right) \rightarrow H_{m_{l+1}}\left(X_{l+1}\right)\right),
$$

and we are done by a simple arrow chasing showing that, if $\alpha \in H_{m_{l}}\left(W_{l}\right)$ goes to $\operatorname{Im}\left(H_{m_{l+1}+2}\left(Y_{l+1}\right) \rightarrow H_{m_{l+1}}\left(X_{l+1}\right)\right)$, then its push forward in $H_{m_{l}}\left(X_{l}\right)$ belongs to $\operatorname{Im}\left(H_{m_{l}+2}\left(Y_{l}\right) \rightarrow H_{m_{l}}\left(X_{l}\right)\right)$. 


\section{ReFERENCES}

[1] Di Gennaro, V. - Franco, D.: Factoriality and Néron-Severi groups, Commun. Contemp. Math. 10, 745-764, 2008.

[2] Di Gennaro, V. - Franco, D.: Monodromy of a family of hypersurfaces, Ann. Scient. Éc. Norm. Sup., $4^{e}$ série, t. 42, 517-529, 2009.

[3] Di Gennaro, V. - Franco, D.: Noether-Lefschetz Theory and Néron-Severi group, Int. J. Math. 23 (2012), 1250004.

[4] Di Gennaro, V. - Franco, D.: Noether-Lefschetz Theory with base locus, Rend. Circ. Mat. Palermo 63, 257-276, 2014.

[5] Dimca, A.: Singularities and Topology of Hypersurfaces, Springer Universitext, New York 1992.

[6] Dimca, A.: Sheaves in Topology, Springer Universitext, 2004.

[7] Flenner, H. - O'Carroll L. - Vogel W.: Joins and intersections, Springer-Verlag, 1999.

[8] Fulton, W.: Intersection theory, Ergebnisse der Mathematik und ihrer Grenzgebiete; 3.Folge, Bd. 2, Springer-Verlag 1984.

[9] Fulton, W.: Young Tableaux, London Mathematical Society Student Texts 35. Cambridge University Press 1997.

[10] Goresky, M. - MacPherson, R.: Stratified Morse Theory, Ergebnisse der Mathematik und ihrer Grenzgebiete; 3.Folge, Bd. 14, Springer-Verlag 1988.

[11] Griffiths, P. - Harris, J.: On the Noether-Lefschetz theorem and some remarks on codimension two cycles, Math. Ann. 271, 31-51, 1985.

[12] Iversen, B: Cohomology of Sheaves Universitext. Springer, 1986.

[13] Lopez, A. F.: Noether-Lefschetz theory and the Picard group of projective surfaces, Mem. Amer. Math. Soc. 89, 1991.

[14] Spanier, E.H.: Algebraic Topology, McGraw-Hill Series in Higher Mathematics, 1966

[15] Voisin, C.: Hodge Theory and Complex Algebraic Geometry, I, Cambridge Studies in Advanced Mathematics 76. Cambridge University Press, 2007.

Università di Roma "Tor Vergata", Dipartimento di Matematica, Via della Ricerca SCIENTIFICA, 00133 Roma, ItAly.

E-mail address: digennar@axp.mat.uniroma2.it

Università di NAPOli "Federico II", Dipartimento di Matematica e Applicazioni "R. CAccioppoli", P.le Tecchio 80, 80125 Napoli, Italy.

E-mail address: davide.franco@unina.it 